\title{
Dermal immune responses against Psoroptes ovis in two cattle breeds and effects of anti-inflammatory dexamethasone treatment on the development of psoroptic mange
}

\author{
Zhenzhen Chen ${ }^{1} \mathbb{B}^{0}$, Edwin Claerebout ${ }^{1 *}$, Koen Chiers ${ }^{2}$, Mathilde Pas $^{3}$, Bart Pardon $^{3}$, Wouter van Mol ${ }^{1}$, \\ Stijn Casaert ${ }^{1}$, Nathalie De Wilde' ${ }^{1}$, Luc Duchateau ${ }^{4}$ and Peter Geldhof ${ }^{1}$
}

\begin{abstract}
Psoroptic mange is a common disease of livestock, caused by Psoroptes ovis. Compared to Holstein-Friesian (HF) cattle, the Belgian Blue (BB) cattle breed is highly susceptible to the infestation. However, the mechanism for this difference is still unclear. To determine the factors responsible for this breed susceptibility, the immune response to $P$. ovis was studied in experimentally infested BB and HF cattle, using clinical signs, histology, immunohistochemical profiling and gene expression analysis of skin biopsies. The mite numbers and lesion area of BB cattle were greater than in $\mathrm{HF}$ during the whole study period. Significant influxes of eosinophils in the epidermis and dermis were detected in comparison with the pre-infestation samples in both breeds, with significantly higher eosinophils in BB at 6 weeks post infestation (wpi). Mast cell numbers were unaffected at all stages of infestation in HF, but were significantly elevated relative to pre-infestation in BB cattle at 2 and 6 wpi. The more pronounced cutaneous eosinophilia and higher IL-4 levels at 6 wpi in BB cattle suggest that a Th2-type immune response is underlying the higher susceptibility of the BB breed. In naturally infested BB cattle, development of the psoroptic mange lesions and eosinophils and CD3+T cell areas were severely depressed after anti-inflammatory treatment with dexamethasone. Together, these results suggest that a stronger Th2-type immune response to $P$. ovis causes the skin lesions in psoroptic mange in BB cattle and that local anti-inflammatory treatment could potentially be an alternative to control the pathology caused by this parasite.
\end{abstract}

Keywords: Psoroptes ovis, immune responses, cattle breeds, anti-inflammatory treatment

\section{Introduction}

Psoroptic mange is a severe disease, which reduces animal welfare and causes financial losses due to performance loss and treatment costs, especially in the sheep and in the beef industry [1-3]. This disease is caused by a non-burrowing ectoparasite, Psoroptes ovis, which lives on the skin surface. The mouthparts of these mites are

*Correspondence: edwin.claerebout@ugent.be

${ }^{1}$ Laboratory of Parasitology, Faculty of Veterinary Medicine, Ghent University, Salisburylaan 133, 9820 Merelbeke, Belgium

Full list of author information is available at the end of the article adapted to the consumption of serous exudate, lymph and red blood cells from the host skin surface [4]. Meanwhile, the mites abrade the stratum corneum and deposit allergens (such as faecal pellets), which cause skin irritation, intense pruritus, and severe allergic dermatitis in livestock [5]. The cutaneous inflammatory response developed by hosts in response to the mites is a serious threat to epidermal integrity [6-8]. The damage in the epidermis is considered as promoting the hypersensitivity reaction in the host to control the mite infestation $[9,10]$. Previous work in sheep demonstrated that a

c) The Author(s) 2020. This article is licensed under a Creative Commons Attribution 4.0 International License, which permits use, sharing, adaptation, distribution and reproduction in any medium or format, as long as you give appropriate credit to the original author(s) and the source, provide a link to the Creative Commons licence, and indicate if changes were made. The images or other third party material in this article are included in the article's Creative Commons licence, unless indicated otherwise in a credit line to the material. If material is not included in the article's Creative Commons licence and your intended use is not permitted by statutory regulation or exceeds the permitted use, you will need to obtain permission directly from the copyright holder. To view a copy of this licence, visit http://creativeco mmons.org/licenses/by/4.0/. The Creative Commons Public Domain Dedication waiver (http://creativecommons.org/publicdomain/ zero/1.0/) applies to the data made available in this article, unless otherwise stated in a credit line to the data. 
reduction of lesion development and mite numbers was caused by suppressing the host immune response [11] However, the relationship between lesion development and immunosuppression in cattle has not been clarified.

Generally, the development of clinical signs in cattle starts at 1 week post infestation [10]. Next, a rapid growth phase sets in with a sharp increase in mite numbers and active lesions that are mainly found at the withers, back and the tail base, but can eventually cover most of the body [3, 12-14]. During this process, pruritus becomes more intense due to the host allergic reaction to the mites, leading to self-trauma behaviour, such as licking and rubbing. Although these actions will make the affected animals comfortable to some degree, mechanical skin abrasion can cause hair loss, skin damage and bleeding wounds [13-15]. All these factors will intensify the local intradermal inflammation, which leads to increased serum extravasation, creating the perfect microclimate for mites to survive. This ideal environment will in turn translate in a further growth of both mite population and skin lesions [10]. Subsequently, the lesion size and mite numbers decrease and, eventually, the clinical signs disappear and the mites are eliminated [2]. However, whether or not animals fully recover from the disease depends on different factors, such as the cattle breed [2, 16]. For instance, natural recovery in Holstein Friesian (HF) cattle is more frequent compared to Belgian Blue (BB) cattle [13].

Previous research suggested a high susceptibility to $P$. ovis infestation in BB cattle under field conditions compared to HF cattle $[2,17]$. Preliminary data suggests that a difference in immune response may contribute to the different breed susceptibility. Previous work revealed that numerous degranulating mast cells and neutrophils were recruited in the skin of Hereford heifer calves in response to $P$. ovis infestation [18], but mast cells did not significantly differ between naturally infested BB and HF cattle [19]. Interestingly, after intradermal injection of Psoroptes cuniculi antigen in cattle of different breeds, an immediate hypersensitivity reaction was observed after $1 \mathrm{~h}$ in HF and BB animals; whereas only in BB cattle a delayed hypersensitivity reaction was recorded after $72 \mathrm{~h}$ $[2,20]$. Moreover, Sarre et al. [19] demonstrated that the up-regulation of IL-17 in the skin of BB cattle was associated with a higher sensitivity to $P$. ovis compared to HF cattle. However, this study was performed in naturally infested animals, which made it impossible to control infestation levels and to determine temporal dynamics of immune responses.

Therefore, in this study, we aimed to compare the development of lesions and the immune response against P. ovis between artificially infested BB and HF cattle, and to investigate the relationship between the inflammatory reaction of the host and lesion development, using an anti-inflammatory dose of dexamethasone in BB cattle. Results were compared with published data from sheep $[6,21]$ and Hereford heifer calves [18], in order to gain insight into critical factors which may be involved in the susceptibility of the $\mathrm{BB}$ cattle breed and the relative resistance of the HF cattle breed.

\section{Materials and methods}

Animal study one: development of psoroptic mange in Belgian Blue and Holstein-Friesian cattle

Nine healthy BB calves and six healthy HF calves (6-7 months old) were included. All calves were free of mite and lice infestation as determined by thorough body inspection and examination of skin scrapings prior to the experiment. All animals were individually stanchioned, with a metal frame around the neck to restrict grooming. P. ovis mites (nymphs and adults) were isolated from naturally infested BB cattle. An area of the left side of the withers $(15 \mathrm{~cm}$ diameter circle) of each animal was shaved with electric clippers, and approximately $400 \sim 500$ mites were placed directly onto the skin of each animal. Mites were prevented from escaping with either a filter paper that was kept in place by tying a rubber band around the animal's chest $(\mathrm{n}=4 \mathrm{BB})$ or with syringes that were cut at the base and glued (super glue, Loctite, Belgium) onto the skin (5 BB and $6 \mathrm{HF}$ ), with each syringe containing $\sim 100$ mites.

Skin biopsies were collected from the infestation site on day 0 (before infestation) and thereafter from the edge of active lesions at weekly intervals from 2 until 6 weeks after infestation. Two skin biopsies were obtained using a disposable $4 \mathrm{~mm}$ biopsy punch (pfm Medical, Germany). Prior to biopsy, the site was subcutaneously injected with a local anaesthetic $(3-4 \mathrm{~mL}, 4 \%$ procaine hydrochloride and $0.0036 \%$ adrenaline tartras, KELA, S.C.-epidural, Belgium). One biopsy was snap-frozen in liquid nitrogen and stored at $-80{ }^{\circ} \mathrm{C}$ until RNA extraction was performed. The other one was stored for $10 \mathrm{~h}$ in $4 \%$ formaldehyde and paraffin-embedded for histology and immunohistochemistry.

The clinical degree of infestation was determined weekly by calculating the percentage of infested body surface (clinical index, CI) for each animal, based on the method of Guillot [22], from day 0 until week 6 p.i. Briefly, all the lesions on the surface of an animal's body were recorded in the grid and then the percentage body surface affected by lesions was calculated. At week $6, P$. ovis mite numbers in active lesions were counted. Skin scrapings were collected from the edges of active lesions or, if lesions regressed during the study, from the area where active lesions were at the study commencement, according to the guidelines of the World Association 
for the Advancement of Veterinary Parasitology [23]. In total three lesion sites per animal were sampled by scraping an area of $9 \mathrm{~cm}^{2}$ per lesion. Samples were examined within $24 \mathrm{~h}$ of collection to identify and count P. ovis mites under the microscope ( $400 \times$ magnification). At the termination of the experiment, all animals were treated topically, twice with a 1-week interval, with amitraz (Taktic $\left.^{\circledR}\right)$ at the recommended dose.

\section{Animal study two: effect of anti-inflammatory treatment on development of psoroptic mange}

Fourteen Belgian Blue calves (1-2 years old) naturally infested with $P$. ovis mites were included in the animal study. Skin scrapings were collected from each animal for mite counts and mite identification on day-7. The CI was determined for each animal by recording the skin lesions (on both sides of the animal) on a silhouette [22]. Animals were randomly assigned to treatment and control group using $\mathrm{CI}$ as stratification factor.

On day 0 , all animals in the treatment group were weighed and injected intramuscularly with dexamethasone (MSD Animal Health, Belgium) at a dose of $0.06 \mathrm{mg} / \mathrm{kg}$ body weight. Control animals were injected with the same volume of physiological saline $(0.9 \%)$. On day 7 and day 14, the treatment was repeated.

All animals were followed for 4 weeks, whereby the CI was determined weekly for each animal as described above. Punch biopsies were taken on day 0 , day 7 and day 28 from the edge of active lesions, following the administration of a local anaesthetic $(3-4 \mathrm{~mL} 4 \%$ procaine hydrochloride and $0.0036 \%$ adrenaline tartrate, KELA, S.C.-epidural, Belgium). The $4 \mathrm{~mm}$ biopsy was immediately fixed in $4 \%$ formaldehyde and paraffin-embedded for histology and immunohistochemistry. At day-7 and day 28 post treatment, $P$. ovis mites in the active lesions were counted, as described above.

All animals were housed together in a pen on straw bedding, and were provided with corn silage, grass silage and water ad libitum and a daily ration of $1.5-2.0 \mathrm{~kg}$ concentrates per animal. All animals were checked weekly for any adverse reactions to the dexamethasone treatment by clinical examination [24] and by ultrasonography (Tringa Linear Vet, Esaote, the Netherlands) to detect (sub)clinical pneumonia. At the end of the animal study all animals were treated topically with amitraz with 2 weeks interval as described above.

\section{Histological and immunohistochemical examination of skin biopsies}

Serial sections $(4-5 \mu \mathrm{m})$ were cut from paraffin embedded biopsies, dewaxed and stained with haematoxylin and eosin (H\&E) for the detection of eosinophils or with Giemsa staining for the detection of mast cells. The number of mast cells and eosinophils was determined at magnification of $400 \times$ using a LEICA light microscope. Therefore, ten photographs were analysed in different dermal layers for each section, i.e. 5 photographs were randomly taken from the epidermis/superficial dermis, and 5 from the deep dermis. Results were expressed as the number of cells per $10^{5} \mu \mathrm{m}^{2}$ tissue surface. The investigator was blinded to the allocated group. An ocular micrometer was used to measure the width of epidermal layer. Five measurements were taken along transects along the skin surface. The thickness was expressed as the mean of 5 measurements at random sites.

Two sections were immunolabelled for CD3 ( $\mathrm{T}$ cells) and CD20 (B cells) based on [19]. In short, skin tissue sections of $4 \mu \mathrm{m}$ were mounted on APES-coated slides, blocked with $\mathrm{H}_{2} \mathrm{O}_{2}$ and stained with polyclonal rabbit anti-human CD3 (Dako, Belgium) or rabbit anti-human CD20 (Sigma-Aldrich, USA) antibodies. T- and B-cells were visualized by adding peroxidase labelled goat antirabbit antibodies (Dako, Belgium), diaminobenzidine tetrahydrochloride (DAB; Dako, Belgium) and by performing a counterstaining with haematoxylin. Pictures at magnification of $400 \times$ using a LEICA light microscope were randomly selected from the epidermis/superficial dermis and the deep dermis as described above. The area percentage of T-cells and B-cells was determined using the Colour Deconvolution plugin in ImageJ [25].

\section{Quantitative real-time polymerase chain reaction (PCR)}

The TissueLyser II instrument (Qiagen) was used to disrupt and homogenize the frozen skin samples. Total RNA was extracted with the RNeasy mini kit (Qiagen) following the manufacturer's protocol with on-column DNase digestion. RNA Quality Index (RQI) was assessed on the Experion automated electrophoresis system (Bio-Rad Laboratories) with the Experion RNA StdSence Analysis kit (Bio-Rad). RNA yield was assessed on a Nano-Drop 2000 spectrophotometer (Thermo Scientific). RNA samples with a RQI $>7.5$ were considered to be of acceptable quality [26]. cDNA was obtained by reverse transcribing $100 \sim 150 \mathrm{ng} / \mu \mathrm{L}$ of purified total RNA using the iScript cDNA synthesis kit (Bio-rad). The cDNA was diluted 1:3 in $\mathrm{dH}_{2} \mathrm{O}$ and stored at $-20^{\circ} \mathrm{C}$.

A quantitative Real-Time PCR (qPCR) approach was used to measure the relative mRNA transcription levels of a selection of genes in the BB and HF calves' skin. The sequences of all the primers that were used can be found in Table 1. qPCR and normalization of the data, based on the housekeeping genes GAPDH and RPS29 for BB and $\mathrm{HF}$, were performed as previously described [19]. Gene transcription levels were evaluated based on relative expression level between the different timepoints. 
Table 1 Primer sequences and amplicon details for BB cattle and HF cattle qPCR

\begin{tabular}{|c|c|c|}
\hline Gene & Accession number & Primer sequence \\
\hline \multirow[t]{2}{*}{ GAPDH } & NM_001034034.1 & F: ACCCAGAAGACTGTGGATGG \\
\hline & & R: CAACAGACACGTTGGGAGTG \\
\hline \multirow[t]{2}{*}{ RPS29 } & BC_102702 & F: GGAGCCATCCGAGAAAATTCG \\
\hline & & R: CAACTTAATGAAGCCGATGTCCTT \\
\hline \multirow[t]{2}{*}{ IL-4 } & NM_173921.2 & F: GCGGACTTGACAGGAATCTC \\
\hline & & R:TCAGCGTACTTGTGCTCGTC \\
\hline \multirow[t]{2}{*}{ IL-5 } & NM_173922.1 & F:TGGTGGCAGAGACCTTGACA \\
\hline & & R: TTCCCATCACCTATCAGCAGAGT \\
\hline \multirow[t]{2}{*}{ IL-6 } & NM_173923.2 & F:TCCTTGCTGCTTTCACACTC \\
\hline & & R: CACCCCAGGCAGACTACTTC \\
\hline \multirow[t]{2}{*}{ |L-8 } & NM_173925.2 & F: GCTGGCTGTTGCTCTCTTGG \\
\hline & & R: GGGTGGAAAGGTGTGGAATGTG \\
\hline \multirow[t]{2}{*}{ IL-10 } & NM_174088.1 & F:TGTATCCACTTGCCAACCAG \\
\hline & & R: CAGCAGAGACTGGGTCAACA \\
\hline \multirow[t]{2}{*}{ IL-13 } & NM_174089.1 & F: GGTGGCCTCACCTCCCCAAG \\
\hline & & R: ATGACACTGCAGTTGGAGATGCTG \\
\hline \multirow[t]{2}{*}{ |L-17 } & NM_001008412.2 & TGAGGACAAGAACTTCCCACAGCA \\
\hline & & TAATCGGTGGGCCTTCTGGAGTTT \\
\hline \multirow[t]{2}{*}{ IL-23A } & NM_0012005688.1 & F: CCCGTATCCAGTGTGAGGAT \\
\hline & & R: AGTATGGAGGCGTGAAGCTG \\
\hline \multirow[t]{2}{*}{ IFN- $-\gamma$} & NM_174086.1 & F:TTCTTGAATGGCAGCTCTGA \\
\hline & & R:TTCTCTTCGGCTTTCTGAGG \\
\hline \multirow[t]{2}{*}{ Foxp3 } & NM_001045933.1 & F: GACAGCACCCTTTCGACTGT \\
\hline & & R: CTCCAGAGATTGCACCACCT \\
\hline \multirow[t]{2}{*}{ Filaggrin } & XM_010826841 & F: GCCCAGTTCTAGACGCTGAC \\
\hline & & R:TCAAGCCAGTGACAGTGAGG \\
\hline \multirow[t]{2}{*}{ Involucrin } & XM_005203832 & F: AAGGTCTTGGGCCAGCACTTG \\
\hline & & R: GATGCTGGGTTGTAACTCCCCCCAC \\
\hline \multirow[t]{2}{*}{ Loricrin } & NM_001113757 & F: CAGTGGATCCGTCTGCCTGGGA \\
\hline & & R: CATGAGAGCGGTAAGCCCATCGAC \\
\hline
\end{tabular}

IL8 (CXCL8) designed based on bovine reference gene sequences from NCBI database. The other primers based on Sarre et al. [19].

\section{Statistical analysis}

All analyses were done using GraphPad Prism 8 (GraphPad Software, San Diego, CA). First, comparing different time points with baseline within group or breed was done in the framework of rank-based repeated measures ANOVA, using Friedman's multiple comparisons technique. Comparing the two groups or breeds at a particular time point was based on the Mann-Whitney $U$ test using Bonferroni multiple comparisons.

\section{Results}

Lesion development and immune response in artificially infested cattle

Before infestation, no skin lesions were observed in the $\mathrm{BB}$ and HF cattle. Active lesions were first observed in both breeds at 2 weeks post infection (wpi). The
CI significantly increased in both breeds from 4 wpi onwards (Figure 1) and at $6 \mathrm{wpi}$, both breeds showed typical clinical signs of psoroptic mange (Additional file 1). Major epidermal pathological changes of $P$. ovis infestation in both breeds included epidermal hyperplasia with marked acanthosis, diffuse hyperkeratosis, irregular rete ridge formation, apoptosis and transudation. Additional pathology in the dermis included oedema, vasculitis and dermal fibrosis (Additional files 2 and 3). Significant influxes of eosinophils, T-lymphocytes and B-lymphocytes in the epidermis/superficial dermis or the deep dermis were detected in comparison with the pre-infestation samples (Figure 2). Mast cell numbers appeared to be unaffected at all stages of infestation in the epidermis and superficial dermis, and were only significantly elevated relative to pre-infestation in the deep dermis of BB cattle (Figure 2). P. ovis infestation was associated with significantly increased transcription of several cytokines and genes related to skin pathology, including interleukin (IL)-4, IL-5 (in HF only), IL-6, IL-8, IL-10, IL-13, IFN- $\gamma$, loricrin (in HF only) and filaggrin (in HF only). No significant changes in IL-17, IL-23, Foxp3 and involucrin transcription were detected in both breeds (Figures 3 and 4), although there was a tendency towards a lower loricrin and filaggrin transcription in BB from 4 wpi onwards (Figure 4).

When both breeds were compared, the clinical index was significantly higher in BB cattle than in HF cattle at 4,5 and 6 wpi. Although the mite numbers did not significantly differ between the two breeds, there was a trend for higher mite numbers in the $\mathrm{BB}$ animals (Figure 1). Compared with HF cattle, significantly higher eosinophil and mast cell infiltration was observed in $\mathrm{BB}$ cattle at $6 \mathrm{wpi}$ in the epidermis/superficial dermis and the deep dermis, respectively (Figure 2 ). In contrast, a larger CD3-positive T-cell area was detected in HF cattle than in BB cattle in the epidermis/superficial dermis and the deep dermis at 5 wpi (Figure 2). Surprisingly, the CD3-positive T-cell area in HF cattle was already significantly higher than in $\mathrm{BB}$ cattle in the epidermis/ superficial dermis and the deep dermis before infestation. Although a larger CD20-positive B-cell area was detected in the epidermis/superficial dermis and the deep dermis in HF cattle than in BB cattle, there was no significant difference between the two breeds. Compared to HF cattle, only the relative transcription levels of IL- 4 were significant higher in BB cattle at $6 \mathrm{wpi}$ (Figure 3). The transcription levels of loricrin and filaggrin were significantly higher in HF cattle than in $\mathrm{BB}$ cattle at $6 \mathrm{wpi}$, and the transcription levels of involucrin were significantly higher in HF cattle before infestation and at 3 and 4 wpi (Figure 4 ). 


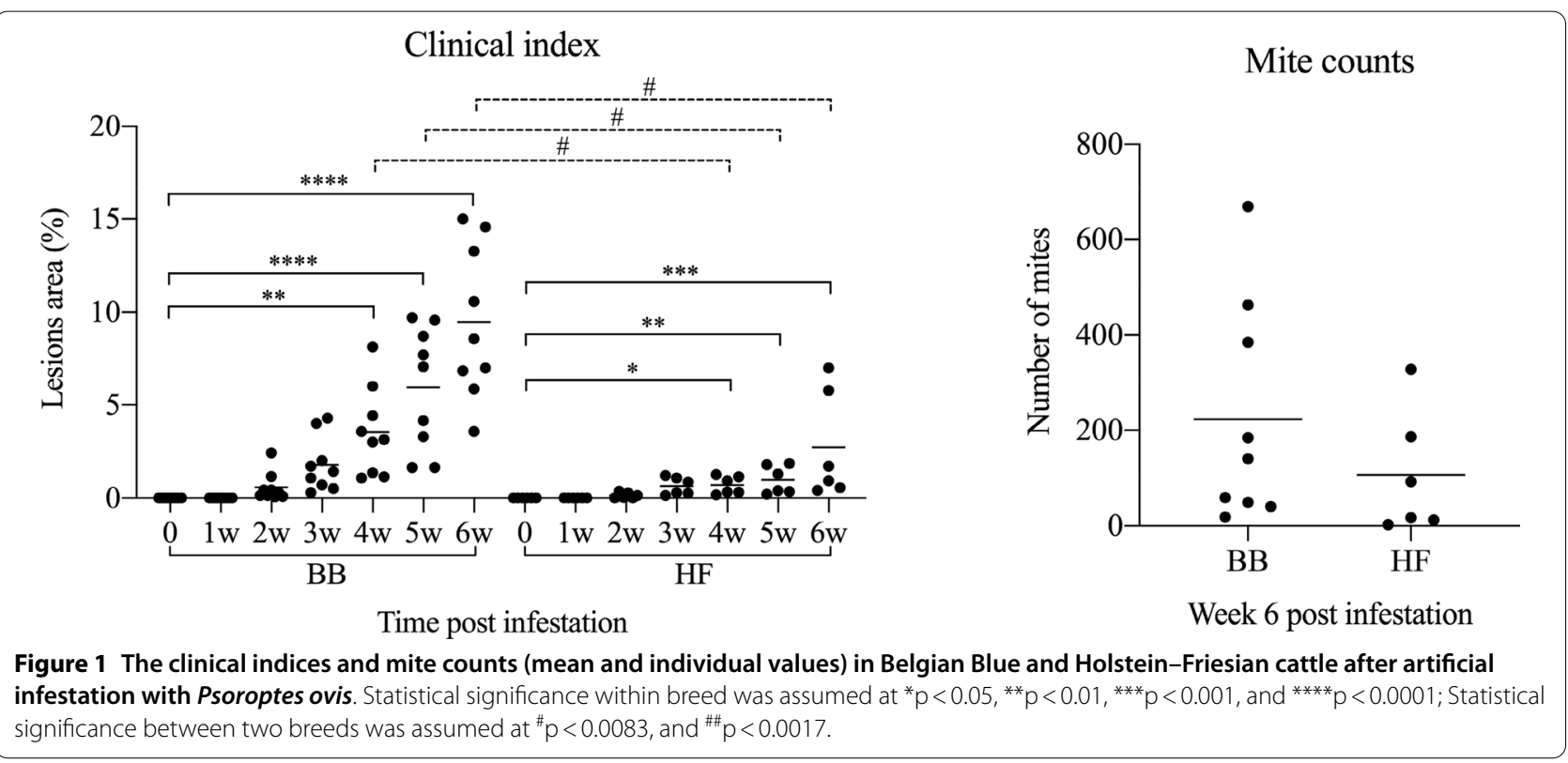

\section{Effect of anti-inflammatory treatment on development of psoroptic mange in BB cattle}

Active lesions in the control animals steadily increased from day 0 onwards. On day 14, three animals in the control group were treated topically with amitraz at the recommended dose for animal welfare reasons. Compared to the control animals, there was a profound reduction in the development of the lesion area by 2 weeks after dexamethasone treatment (day 14), and this difference was maintained until termination of the study (day 28), when the CI was decreased by $90 \%$ compared to the control group. Mean mite counts were reduced by $56 \%$ in the treated group compared with the control animals on day 28 , but the number of mites was not significantly different between the two groups (Figure 5).

Eosinophil numbers in the epidermis/superficial dermis and in the deep dermis were significantly depressed in the treatment group compared to the control group on day 7 and day 28 . The number of mast cells did not differ between both groups. The CD3-positive T-cell areas were significantly reduced on day 28 after dexamethasone treatment. Although the mean CD20-positive B-cell area in the treatment group was lower than in the control group on day 28, there was no significant difference between the two groups (Figure 6).

\section{Discussion}

Individual and breed differences in susceptibility to infestation with $P$. ovis and the related Sarcoptes scabiei (S. scabiei) mites have been associated with pathological changes and immune responses in various host species $[2,27-29]$. The available studies suggest that the complex relationship between the host and the parasite at the skin interface results in a balance between the immune responses and clinical signs [30-32], yet many questions remain unclear.

The HF cattle breed is less susceptible to $P$. ovis infestation in comparison to BB cattle in the field [2,33]. Given that the immune response is at least partially responsible for the lesion development, a possible reason for the difference of lesion development between BB and HF cattle could be related to different immune responses after $P$. ovis infestation. In the first animal study, the dermal immune response in $\mathrm{BB}$ cattle and HF cattle during an experimental $P$. ovis infestation was compared, in an attempt to identify a potential cause of the severe manifestation of psoroptic mange in the BB cattle breed compared to HF cattle.

The significant difference between the clinical indices of both breeds confirmed that BB cattle were more susceptible to the induced $P$. ovis infestation than HF. A previous study also showed that $\mathrm{BB}$ cattle were more susceptible to artificial $P$. ovis infestation than HF cattle, but the differences were not significant [2], which may be due to the small sample size. Apart from these quantitative differences, the clinical picture and histological changes in HF cattle were similar to BB cattle and were characterized by erythematous scaly papules and plaque formation and infiltration of inflammatory cells into the epidermis and dermis. Significant influxes of eosinophils in the epidermis/superficial dermis and the deep dermis were detected in comparison with the pre-infestation in BB cattle from 3 wpi onwards, but in HF cattle only at 5 wpi. Moreover, the number 
Eosinophils - epidermis/superficial dermis

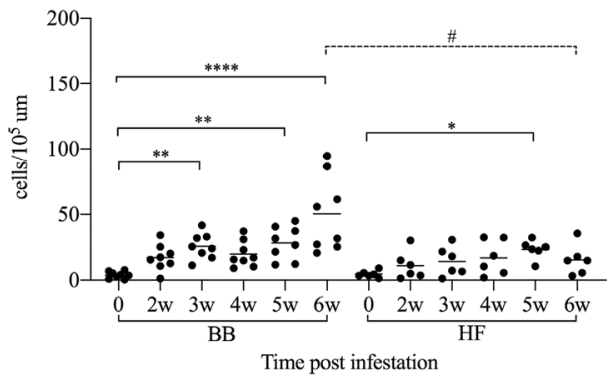

Mast cells - epidermis/superficial dermis

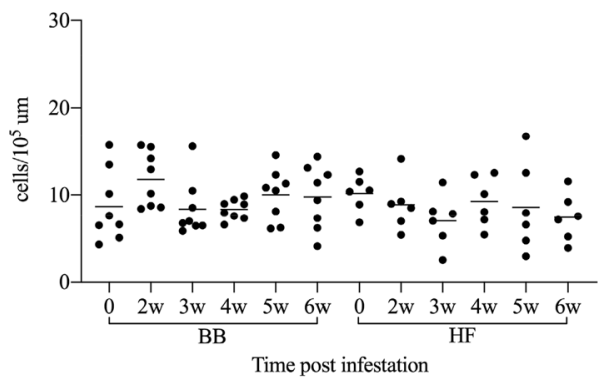

CD3-epidermis/superficial dermis

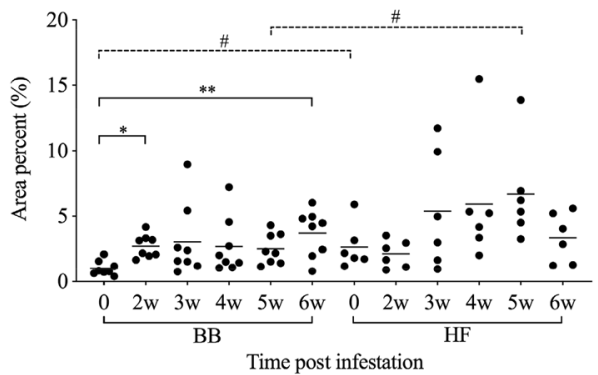

CD20 - epidermis/superficial dermis

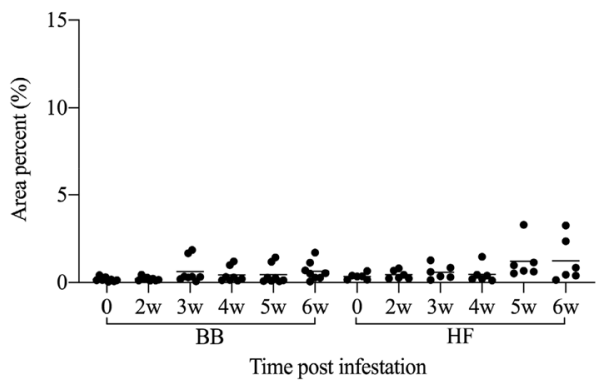

Eosinophils - deep dermis

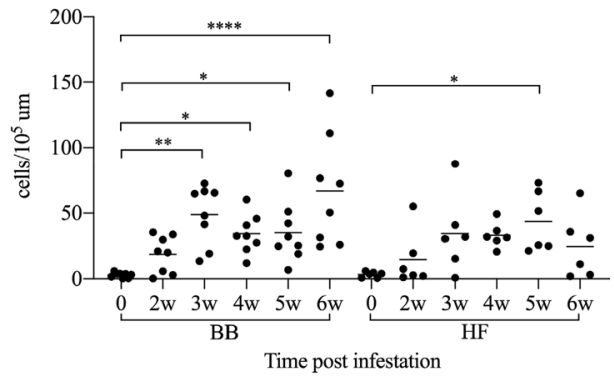

Mast cells - deep dermis

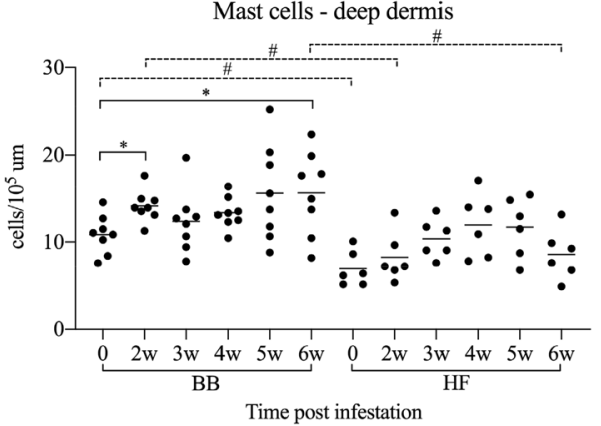

CD3-deep dermis

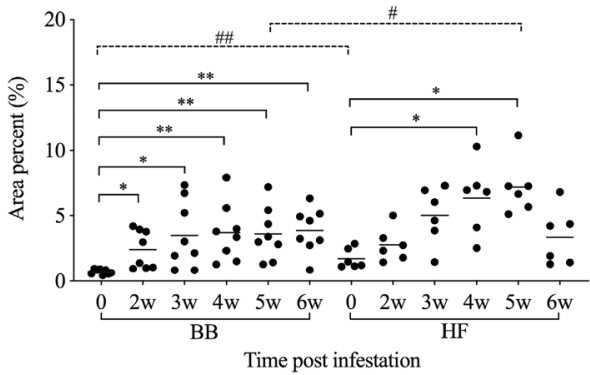

CD20 - deep dermis

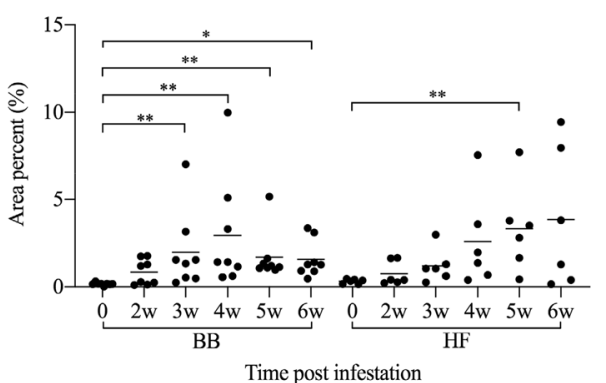

Figure 2 Histological cell counts (mean and individual values) in the epidermis/superficial dermis and the deep dermis of skin biopsies in Belgian Blue and Holstein-Friesian cattle at different time points after artificial infestation with Psoroptes ovis. The results are presented as number of eosinophils and mast cells per $10^{5} \mathrm{\mu m}^{2}$ and as area percent of CD3 + (T-cells) and CD20 + (B-cells). Statistical significance within breed was assumed at ${ }^{*} p<0.05,{ }^{* *} p<0.01,{ }^{* *} p<0.001$, and ${ }^{* * *} p<0.0001$; statistical significance between two breeds was assumed at ${ }^{\#} p<0.0083$, and $\# \mathrm{p}<0.0017$. Only 8 BB cattle were included in the statistical analysis of the cell counts as from one animal missing values were obtained at 4 wpi. 


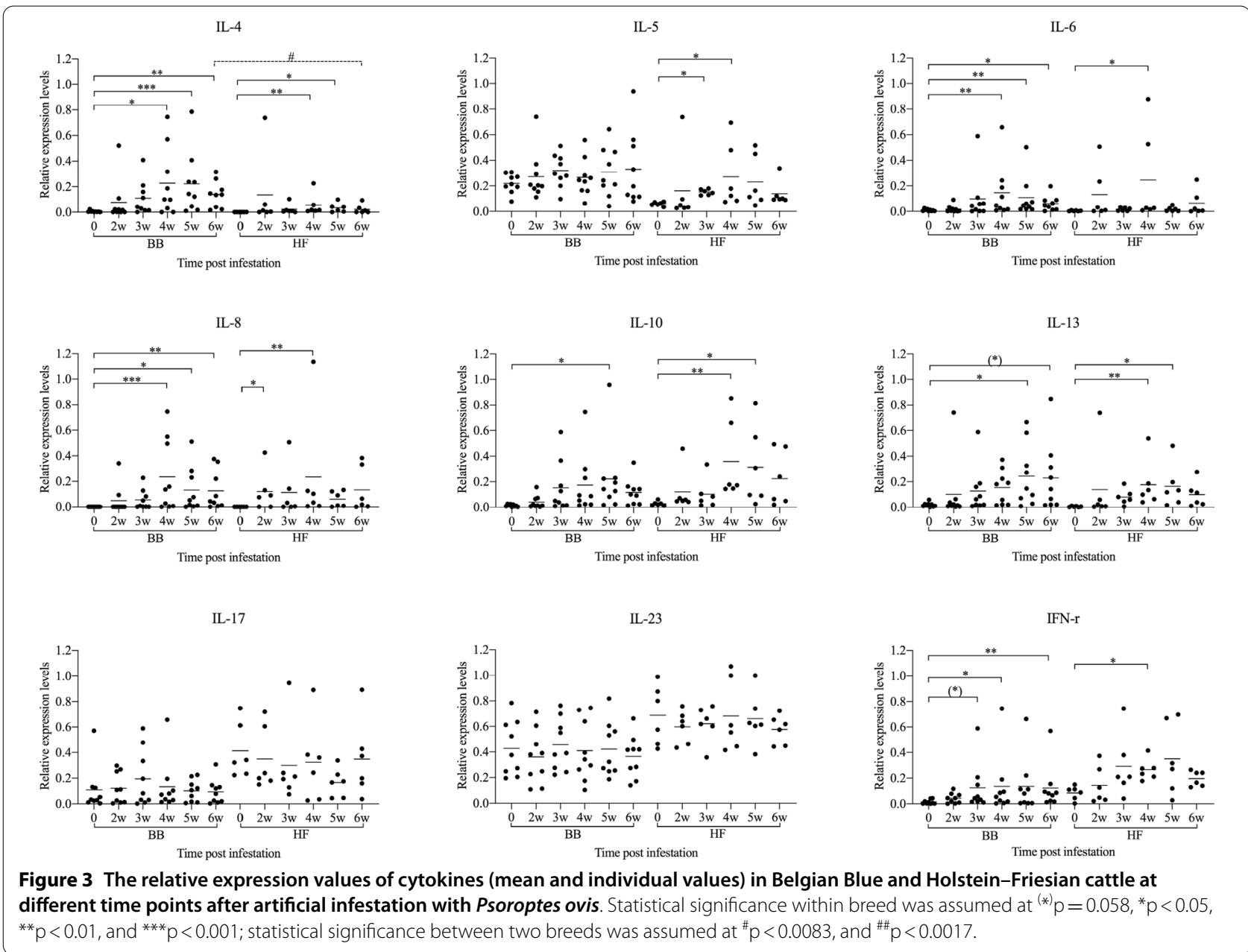

of eosinophils in BB cattle was significantly higher and increased steadily until the end of the experiment, whereas eosinophils in HF cattle peaked at 5 wpi and started to decline thereafter. However, the epidermal differentiation complex (EDC) genes, such as filaggrin, loricrin and involucrin were significantly upregulated in HF cattle, while the transcription of filaggrin and loricrin was slightly downregulated (NS) in BB cattle. Previous studies suggested that EDC genes could be down-regulated through the suppressive action of Th2 cytokines, such as IL-4 and IL-13 [34, 35]. Together with the more pronounced cutaneous eosinophilia and higher IL-4 levels at 6 wpi in BB cattle, this suggests that a Th2-type immune response is underlying the more severe manifestation in the BB breed. Similarly, a (pro-)inflammatory response, evolving into a Th2-type immune response was previously observed in $P$. ovis infestations in sheep $[6,36]$. In sheep, the maximal number of eosinophils was observed at 9 wpi [36]. Possibly, differences between the two cattle breeds may become more pronounced later in the infestation.
The CD3-positive T-cell area in HF cattle was significantly higher than in BB cattle. Since no further T-lymphocyte markers were used in our experiment, the T-cell population could not be subtyped. We hypothesized that $\mathrm{a}$ (proportion of) the larger population of $\mathrm{T}$ lymphocytes in HF cattle could be regulatory T-cells that are able to suppress the immune responses [37]. However, this hypothesis was not supported by the transcription levels of IL-10 and Foxp3, which were not significantly different between the two breeds. An increased Th2/Th17 immune response was associated with increased susceptibility to S. scabiei infestations in humans and pigs [27, 29,38 ] and in BB cattle naturally infested by $P$. ovis [19]. Although IL-17 levels were not significantly different within both breeds in our study, the expression of IL-17 was slightly increased in BB cattle and was decreased in HF cattle in comparison with pre-infestation. This is similar to the results of Sarre et al. [19]. Previous work in pigs with S. scabiei infestations showed an upregulation of IL-17 from 4 wpi onwards, whereas the peak expression was observed at 8 wpi [29]. Also, an increased IL-17 
Foxp3

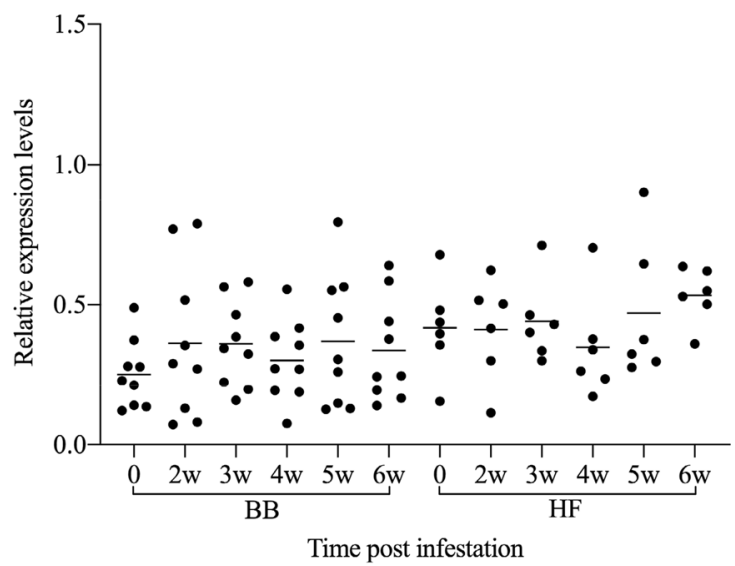

Loricrin

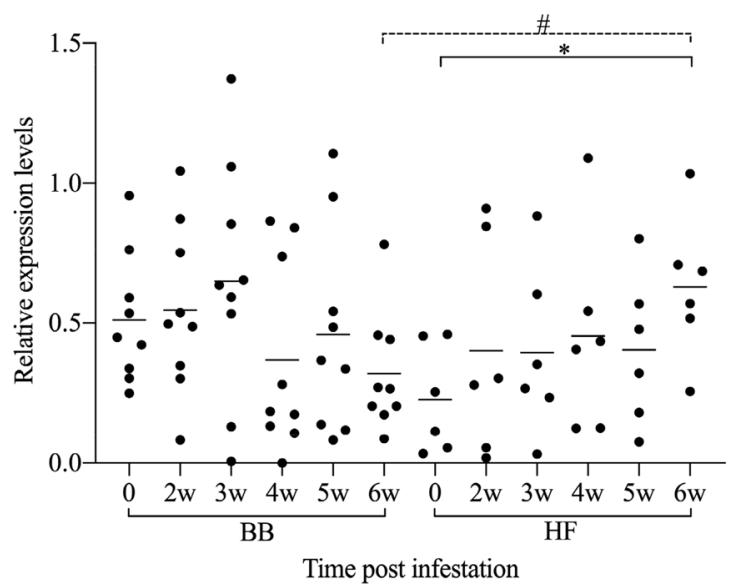

Involucrin

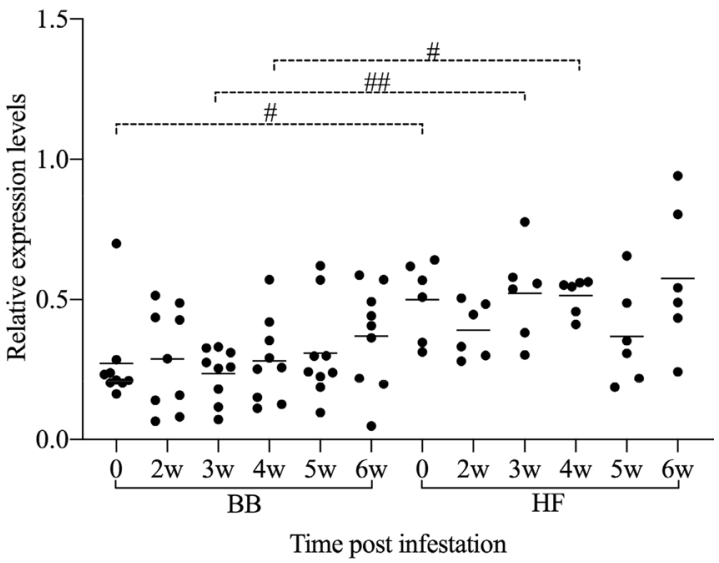

Filaggrin

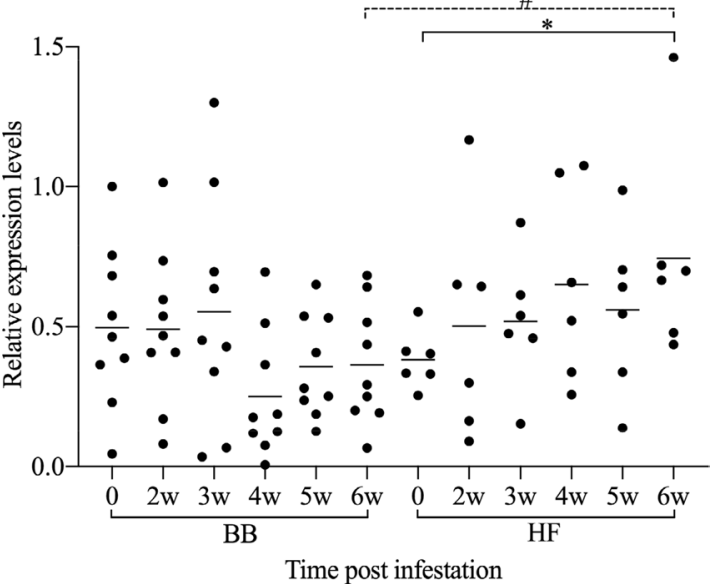

Figure 4 The relative transcription levels of genes related to pathology (mean and individual values) in Belgian Blue and HolsteinFriesian cattle at different time points after artificial infestation with Psoroptes ovis. Statistical significance within breed was assumed at ${ }^{*} p<0.05$; statistical significance between two breeds was assumed at ${ }^{\#} p<0.0083$, and ${ }^{\# \#} p<0.0017$.

Clinical index

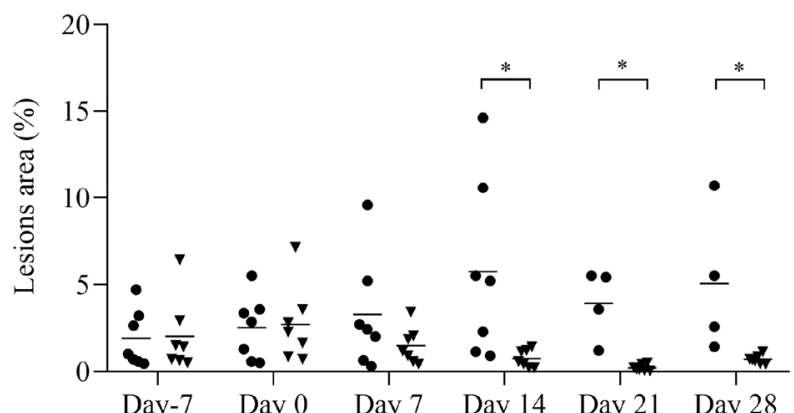

Mite counts

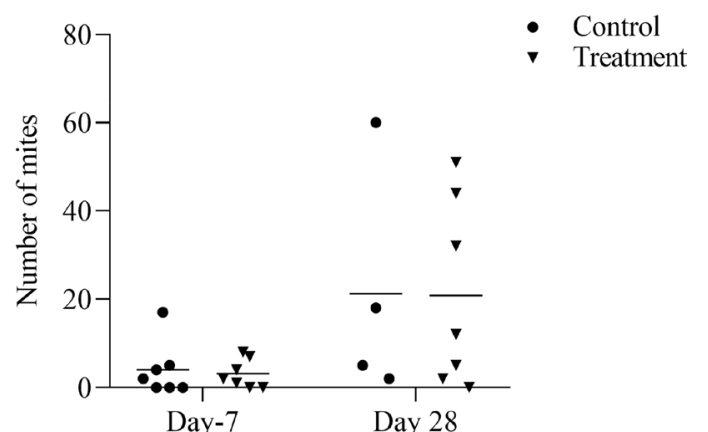

Day 28

Figure 5 The clinical index and mite counts (mean and individual values) in Belgian Blue cattle that were treated with dexamethasone on days 0,7 and 14 (Treatment group) or physiological saline (Control group). Statistical significance was assumed at ${ }^{*} p<0.01$ and ${ }^{* *} p<0.002$ for clinical index; statistical significance was assumed at ${ }^{*} p<0.05$ for mite counts). On day 14 , three animals in the control group were treated topically with amitraz at the recommended dose for animal welfare reasons. 
Eosinophils-epidermis/superficial dermis

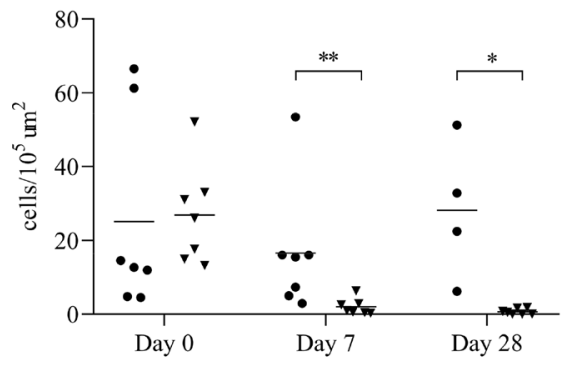

Mast cells-epidermis/superficial dermis

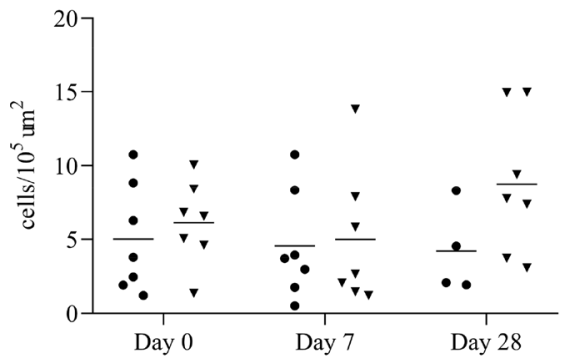

CD3-epidermis/superficial dermis

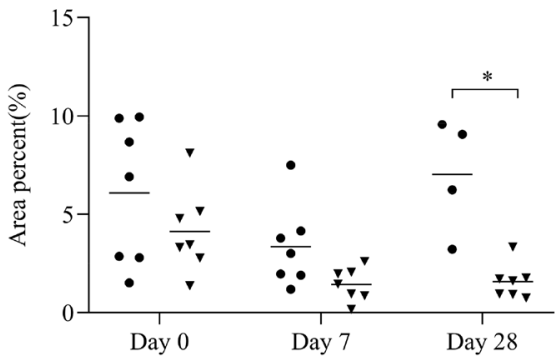

CD20-epidermis/superficial dermis

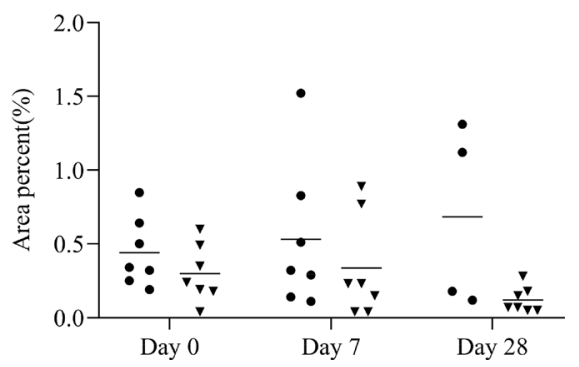

Eosinophils-deep dermis

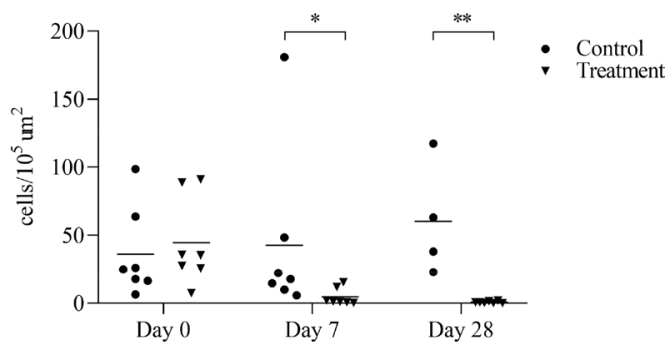

Mast cells-deep dermis

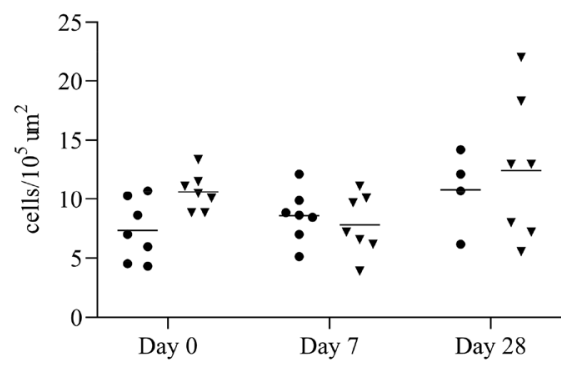

CD3-deep dermis

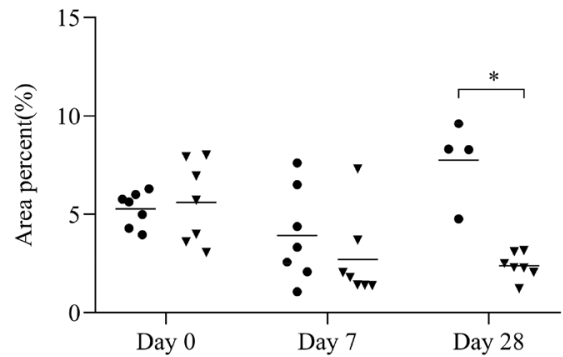

CD20-deep dermis

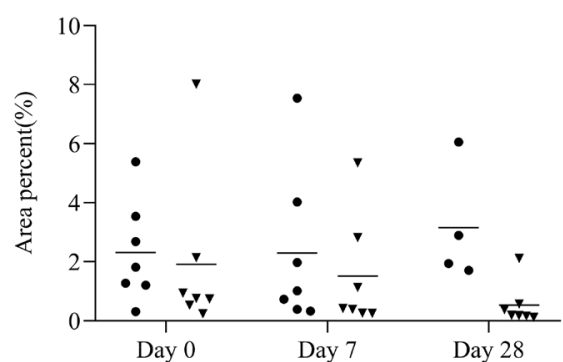

Figure 6 Histological cell counts (mean and individual values) in the epidermis/superficial dermis and the deep dermis of skin biopsies in Belgian Blue cattle that were treated with dexamethasone on days 0,7 and 14 (Treatment group) or with physiological saline (Control group). The results are presented as mean number of eosinophils and mast cells per $10^{5} \mu \mathrm{m}^{2}$ and as area percent of CD3+(T-cells) and CD20+ (B-cells). Statistical significance was assumed at ${ }^{*} p<0.017$ and ${ }^{* *} p<0.003$. On day 14 , three animals in the control group were treated topically with amitraz $\left(\operatorname{Taktic}^{\circledR}\right)$ at the recommended dose for animal welfare reasons. 
was detected at $15 \mathrm{wpi}$ [38]. These results suggest that the Th17 immune response is related to the infestation time. Therefore, it would be interesting to investigate whether or not IL-17 transcription would further diverge between $\mathrm{BB}$ cattle and HF cattle when the infestation time is extended.

Since the higher susceptibility of BB cattle was shown to be associated with a stronger Th2-type response, our second objective was to investigate the causal relationship between the host's immune response and the development of skin lesions. Treatment of BB cattle with dexamethasone induced a profound suppression of lesion development, but no significant reduction in mite numbers compared to the control group. Although in the study of Huntley et al. [11], anti-inflammatory treatment of sheep with Cyclosporin A led to significantly depressed lesion development and almost totally reduced mite numbers compared to the normal course of infection, previous studies showed that there is no strong relationship between the clinical index and mite numbers in cattle [2, 33]. Skin healing was accompanied by significantly reduced eosinophils and T-lymphocytes in the skin biopsies, which is similar with observations in sheep scab [11, 39]. In sheep, no difference was observed in the population of mast cells/basophils between control animals and animals treated with an anti-inflammatory drug [11], which is in accordance with the results of our study. Clearly, an inflammatory response is related to the development of psoroptic mange lesions in both sheep and cattle, but mast cells do not seem to be important in this process in cattle.

\section{Conclusions}

The development of skin lesions in psoroptic mange in cattle is associated with the host immune response, as lesions development was reversed by anti-inflammatory treatment. The higher susceptibility of $\mathrm{BB}$ cattle compared to the HF breed appears to be due to a more pronounced Th2-type (and potentially Th-17) immune response in $\mathrm{BB}$. A stronger T-cell response was observed in the more resistant HF breed, but it is as yet unclear with T-cell subtypes are involved. Identification of these T-cell subsets and extending the duration of the artificial infestations could further clarify the difference in the immune responses between both breeds. Local antiinflammatory treatment could potentially be an alternative method for controlling the pathology caused by a $P$. ovis infestation.

\section{Supplementary information}

The online version contains supplementary material available at https://doi. org/10.1186/s13567-020-00874-x.
Additional file 1. Clinical appearance and close-up view of Belgian Blue $(A, C)$ and Holstein-Friesian cattle $(B, D)$ infested with Psoroptes ovis at 6 weeks post-infestation, respectively. The yellow circles indicate active lesions on the animals' skin.

Additional file 2. Representative histology of skin lesions at 6 weeks post infestation with Psoroptes ovis in Belgian Blue cattle (A: $x$ 100; B: $\times \mathbf{4 0 0 )}$ ). A: Parakeratosis (filled arrow), dermal oedema and disruption of collagen bundles (dashed line arrows). B: Intercellular oedema, disruption of intercellular bridges and hydeopic degeneration of cells in the epidermis (filled arrows)

Additional file 3. The width of the epidermis at different time points in Belgian Blue (BB) and Holstein-Friesian (HF) cattle after artificial infestation with Psoroptes ovis (mean and individual values). ${ }^{*} p<0.05,{ }^{* *} p<0.01$, and ${ }^{* * *} p<0.001$. Only 7 BB cattle were included in the statistical analysis as from two animals a missing value was obtained at 3 and 4 weeks post-infestation.

\section{Abbreviations}

BB: Belgium Blue; Cl: clinical index; HF: Holstein-Friesian; IL-: interleukin-; P. ovis: Psoroptes ovis; S. scabiei: Sarcoptes scabiei; Wpi: weeks post infestation; Th: T-helper cell.

\section{Acknowledgements}

The authors would like to thank the farmers involved in this study and Lobke De Bels, Delphine Ameye, and Joachim Christiaens for their excellent technical assistance. Zhenzhen Chen is supported by the China Scholarship Council (CSC number 201606910041).

\section{Authors' contributions}

ZC participated in the design of the study, manuscript writing, data analysis and performed the experiments; EC participated in the design of the study and manuscript writing; KC participated in the analysis of histopathological images; LD and PG participated in the analysis of APCR; MP, BP, WM, SC, and NW participated in the animal studies. All authors read and approved the final manuscript.

\section{Funding}

Not applicable.

\section{Availability of data and materials}

All data generated or analysed during this study are included in this published article and its Additional files.

\section{Ethics approval and consent to participate}

Animal study one was approved by the Ethical Committee of the Faculties of Veterinary Medicine and Bioengineering Sciences of Ghent University (EC2019/64); Animal study two was approved by the Ethical Committee of the Faculties of Veterinary Medicine and Bioengineering Sciences of Ghent University (EC2019/103), Ghent, Belgium and by the Department of Environment of the Flemish government, Belgium (LA1400086-Veldproef 2020V05).

\section{Consent for publication}

Not applicable.

\section{Competing interests}

The authors declare no conflict of interest.

\section{Author details}

${ }^{1}$ Laboratory of Parasitology, Faculty of Veterinary Medicine, Ghent University, Salisburylaan 133, 9820 Merelbeke, Belgium. ${ }^{2}$ Department of Pathology, Bacteriology and Poultry Diseases, Faculty of Veterinary Medicine, Ghent University, Salisburylaan 133, 9820 Merelbeke, Belgium. ${ }^{3}$ Department of Large Animal Internal Medicine, Faculty of Veterinary Medicine, Ghent University, Salisburylaan 133, 9820 Merelbeke, Belgium. ${ }^{4}$ Biometrics Research Center, Faculty of Veterinary Medicine, Ghent University, Salisburylaan 133, 9820 Merelbeke, Belgium. 
Received: 15 September 2020 Accepted: 3 December 2020

Published online: 04 January 2021

\section{References}

1. Rehbein S, Visser M, Winter R, Trommer B, Matthes H-F, Maciel A, Marley S (2003) Productivity effects of bovine mange and control with ivermectin. Vet Parasitol 114(4):267-284

2. Losson B, Lonneux J-F, Lekimme M (1999) The pathology of Psoroptes ovis infestation in cattle with a special emphasis on breed difference. Vet Parasitol 83(3):219-229

3. Mitchell S (2010) Psoroptic mange: a severe threat to UK cattle. Cattle Practice 18(3):146-150

4. DeLoach JR, Wright FC (1981) Ingestion of rabbit erythrocytes containing 51Cr-labeled hemoglobin by Psoroptes spp. (Acari: Psoroptidae) that originated on cattle, mountain sheep, or rabbits. J Med Entomol 18(4):345-348

5. Mathieson B, Lehane M (2002) Ultrastructure of the alimentary canal of the sheep scab mite, Psoroptes ovis (Acari: Psoroptidae). Vet Parasitol 104(2):151-166

6. Burgess ST, Frew D, Nunn F, Watkins CA, McNeilly TN, Nisbet AJ, Huntley JF (2010) Transcriptomic analysis of the temporal host response to skin infestation with the ectoparasitic mite Psoroptes ovis. BMC Genomics 11(1):624

7. Van den Broek A, Huntley J (2003) Sheep scab: the disease, pathogenesis and control. J Comp Pathol 128(2-3):79-91

8. Hamilton K, Nisbet A, Lehane M, Taylor M, Billingsley PF (2003) A physiological and biochemical model for digestion in the ectoparasitic mite, Psoroptes ovis (Acari: Psoroptidae). Int J Parasitol 33(8):773-785

9. Bates P (2012) External parasites of small ruminants. A practical guide to their prevention and control. CABI, Wallingford

10. Stromberg PC, Fisher W (1986) Dermatopathology and immunity in experimental Psoroptes ovis (Acari: Psoroptidae) infestation of naive and previously exposed Hereford cattle. Am J Vet Res 47(7):1551-1560

11. Huntley JF, van den Broek A, Machell J, Mackellar A, Pettit D, Meikle L, Barcham G, Meeusen EN, Smith D (2005) The effect of immunosuppression with cyclosporin A on the development of sheep scab. Vet Parasitol 127(3-4):323-332

12. Stromberg P, Guillot $F$ (1987) Hematology in the regressive phase of bovine psoroptic scabies. Vet Pathol 24(5):371-377

13. Bates $P$ (1998) Bovine mange in great Britain. Cattle Pract 6(1):53-57

14. Pouplard L, Losson B, Detry M, Hollanders W (1990) Les gales bovines. Ann Med Vet 134:531-539

15. Mitchell E, Jones J, Foster A, Millar M, Milnes A, Williams J (2011) Clinical features of psoroptic mange in cattle in England and Wales. Vet Rec. 170:100565

16. Roberts IH, Meleney WP (1971) Variations among strains of Psoroptes ovis (Acarina: Psoroptidae) on sheep and cattle. Ann Entomol Soc Am 64(1):109-116

17. Sarre C, De Bleecker K, Deprez P, Levecke B, Charlier J, Vercruysse J, Claerebout E (2012) Risk factors for Psoroptes ovis mange on Belgian Blue farms in Northern Belgium. Vet Parasitol 190(1):216-221

18. Stromberg P, Guillot F (1989) Pathogenesis of psoroptic scabies in Hereford heifer calves. Am J Vet Res 50(4):594-601

19. Sarre C, González-Hernández A, Coppernolle S, Grit R, Grauwet K, Meulder F, Chiers K, den Broeck W, Geldhof P, Claerebout E (2015) Comparative immune responses against Psoroptes ovis in two cattle breeds with different susceptibility to mange. Vet Res 46(1):131

20. Losson B, Detry-Pouplard M, Pouplard L (1988) Haematological and immunological response of unrestrained cattle to Psoroptes ovis, the sheep scab mite. Res Vet Sci 44(2):197-201

21. Burgess ST, McNeilly TN, Watkins CA, Nisbet AJ, Huntley JF (2011) Host transcription factors in the immediate pro-inflammatory response to the parasitic mite Psoroptes ovis. PLoS ONE 6(9):e24402
22. Guillot FS (1981) Population increase of Psoroptes ovis (Acari: Psoroptidae) on stanchioned cattle during summer. J Med Entomol 18(1):44-47

23. Vercruysse J, Rehbein S, Holdsworth P, Letonja T, Peter R (2006) World Association for the Advancement of Veterinary Parasitology (WAAVP) guidelines for evaluating the efficacy of acaricides against (mange and itch) mites on ruminants. Vet Parasitol 136(1):55-66

24. Dirksen G, Gründer H-D, Stöber M (1990) Die klinische Untersuchung des Rindes, 3rd edn. Verlag Paul Parey, Berlin

25. Ross J (2014) Using the color deconvolution plugin in ImageJ. Biomed Imaging Res Unit: 1-11. Available at:https://www.fmhs.auckland.ac.nz/ assets/fmhs/sms/biru/docs/Using\%20Colour\%20Deconvolution \%20in\%20lmageJ.pdf. Accessed 4 Sept 2014

26. Denisov V, Strong W, Walder M, Gingrich J, Wintz H (2008) Development and validation of RQI: an RNA quality indicator for the Experion ${ }^{\mathrm{TM}}$ automated electrophoresis system. Electrophoresis tech note 5761. Available at: http://www.gene-quantification.com/Bio-Rad-bulletin-5761.pdf. Accessed19 Jan 2016

27. Walton S (2010) The immunology of susceptibility and resistance to scabies. Parasite Immunol 32(8):532-540

28. Walton SF, Oprescu FI (2013) Immunology of scabies and translational outcomes: identifying the missing links. Curr Opin Infect Dis 26(2):116-122

29. Mounsey KE, Murray HC, Bielefeldt-Ohmann H, Pasay C, Holt DC, Currie BJ, Walton SF, McCarthy JS (2015) Prospective study in a porcine model of Sarcoptes scabiei indicates the association of Th2 and Th17 pathways with the clinical severity of scabies. PLoS Negl Trop Dis 9(3):e0003498

30. van den Broek A (2007) Pathophysiology of ectoparasitic disease. UK Vet Companion Anim 12(4):63-68

31. Arlian LG, Morgan MS (2017) A review of Sarcoptes scabiei: past, present and future. Parasites Vectors 10(1):297

32. van den Broek A, Burgess ST (2013) Psoroptes Ovis. In: Noli C, Foster A, Rosenkrantz W (eds) Veterinary Allergy. John Wiley \& SonsLtd, Chichester, pp 402-410

33. Lonneux J-F, Nguyen T, Detry J, Farnir F, Losson B (1998) The relationship between parasite counts, lesions, antibody titres and daily weight gains in Psoroptes ovis infested cattle. Vet Parasitol 76(1-2):137-148

34. Kim BE, Leung DY, Boguniewicz M, Howell MD (2008) Loricrin and involucrin expression is down-regulated by Th2 cytokines through STAT-6. Clin Immunol 126(3):332-337

35. Howell MD, Kim BE, Gao P, Grant AV, Boguniewicz M, De Benedetto A, Schneider L, Beck LA, Barnes KC, Leung DY (2009) Cytokine modulation of atopic dermatitis filaggrin skin expression. J Allergy Clin Immunol 124(3):R7-R12

36. Van Den Broek A, Else R, Huntley J, Machell J, Taylor M, Miller H (2004) Early innate and longer-term adaptive cutaneous immunoinflammatory responses during primary infestation with the sheep scab mite, Psoroptes ovis. J Comp Pathol 131(4):318-329

37. Arlian LG, Morgan MS, Paul CC (2014) Evidence that scabies mites (Acari: Sarcoptidae) influence production of interleukin-10 and the function of T-regulatory cells (Tr1) in humans. J Med Entomol 43(2):283-287

38. Liu X, Walton S, Murray HC, King M, Kelly A, Holt DC, Currie BJ, McCarthy JS, Mounsey K (2014) Crusted scabies is associated with increased IL-17 secretion by skin T cells. Parasite Immunol 36(11):594-604

39. Den Broek V (2000) Cutaneous and systemic responses during primary and challenge infestations of sheep with the sheep scab mite, Psoroptes ovis. Parasite Immunol 22(8):407-414

\section{Publisher's Note}

Springer Nature remains neutral with regard to jurisdictional claims in published maps and institutional affiliations. 